\title{
Propuesta para un modelo descriptivo y analítico de las narrativas transmedia no- ficcionales
}

\section{Proposal for a descriptive and analytical model of non-fictional transmedia narratives.}

DOI: $10.46932 / \mathrm{sfjdv} 2 \mathrm{n} 2-038$

Received in: january 1st, 2020

Accepted in: March 30th, 2020

\section{Martín Eugenio Astigueta}

Master degree

Current Institution: Facultad de Ciencias de la Educación y de la Comunicación Social (FCECS), Universidad del Salvador (USAL)

Full adress: Perón 3801, 4B, Ciudad Autónoma de Buenos Aires, Argentina. ZIP code: C1198AAR

E-mail: mastigueta@gmail.com

\section{RESUMEN}

El objetivo general de este trabajo es indagar sobre la transmedialidad entendida en cuanto fenómeno discursivo asociado a relatos no-ficcionales como el periodismo y los documentales interactivos. En lo particular, se analizó diferentes piezas informativas transmedia a los fines de detectar la presencia de elementos comunes y recurrentes que permitieran realizar aportes para una sistematización y tipificación de las narrativas transmedia no-ficcionales.

Desde su diseño, esta investigación constó de tres fases sucesivas. En la primera se procedió a integrar y sintetizar las seis tipologías de las narrativas transmedia más difundidas a hasta el 2016, las de: Henry Jenkins, Laurel Ryan, Jeff Gómez, Carlos Scolari, la del MIT Open Documentary Lab e IDFA DocLab, $\mathrm{y}$, la del colectivo de investigadores, realizadores y autores firmantes del Manifiesto Transmedia. De esta forma, se obtuvo una tipología resultante constituida por un total de 12 principios dentro de los cuales se pueden englobar los elementos presentes en las otras propuestas.

En las subsiguientes fases, se buscó someter la tipología resultante a diferentes análisis cuantitativos y cualitativos para evaluar su efectividad teórico-empírica para la descripción de diferentes narrativas transmedia no-ficcionales. Para ello, se construyó una base de datos conformada por 216 casos de estudio constituido por documentales web y otras piezas interactivas, que fueron analizados con la asistencia del programa informático SPSS. Durante la segunda y tercera fase, se seleccionaron diferentes casos de estudio, y se los sometió a diferentes análisis semióticos y narratológicos, siguiendo los principios de la tipología propuesta.

Los resultados obtenidos durante las diferentes etapas de análisis permitieron comprobar el alto grado de efectividad de la tipología construida para la descripción y análisis de las piezas transmedia no-ficcionales. Se considera que este trabajo realiza una contribución a un campo que se mantiene poco estudiado a nivel académico, pero que a nivel profesional evidencia un importante grado de desarrollo.

\footnotetext{
ABSTRACT

The general objective of this paper is to investigate transmediality understood as a discursive phenomenon associated with non-fictional narratives such as journalism and interactive documentaries. In particular, different transmedia informative pieces were analyzed in order to detect the presence of common and recurrent elements that would allow contributions to be made for a systematization and typification of non-fictional transmedia narratives.
} 
From its design, this research consisted of three successive phases. In the first one, we proceeded to integrate and synthesize the six typologies of the most widespread transmedia narratives as of 2016, those of: Henry Jenkins, Laurel Ryan, Jeff Gómez, Carlos Scolari, that of the MIT Open Documentary Lab and IDFA DocLab, and, that of the collective of researchers, filmmakers and authors who signed the Transmedia Manifesto. This resulted in a typology consisting of a total of 12 principles within which the elements present in the other proposals can be included.

In the subsequent phases, the resulting typology was subjected to different quantitative and qualitative analyses in order to evaluate its theoretical-empirical effectiveness for the description of different nonfictional transmedia narratives. To this end, a database of 216 case studies consisting of web documentaries and other interactive pieces was constructed and analyzed with the assistance of SPSS software. During the second and third phases, different case studies were selected and subjected to different semiotic and narratological analyses, following the principles of the proposed typology.

The results obtained during the different stages of analysis allowed us to verify the high degree of effectiveness of the typology constructed for the description and analysis of non-fictional transmedia pieces. It is considered that this work makes a contribution to a field that remains little studied at the academic level, but that at the professional level shows an important degree of development.

\section{INTRODUCCIÓN}

Este trabajo es el resultado de un programa de investigación constituido por tres trabajos sucesivos orientados a explorar a través de diferentes análisis cuantitativos y cualitativos realizados entre 2014 y 2017 la posibilidad de incluir a ciertos relatos multimedia no-ficcionales, como los documentales interactivos y otras producciones periodísticas multiplataforma, dentro del análisis de las denominadas "narrativas transmedia".

Así, durante su primera etapa, titulada "Reflexiones para una tipología de las narrativas transmediáticas no-ficcionales", se procedió a integrar y sintetizar las seis tipologías más extendidas hasta el 2016: la de Henry Jenkins (2009), Laurel Ryan (2000), Jeff Gomez (2007), Carlos Scolari (2013c), la del MIT Open Documentary Lab e IDFA DocLab (2012) ${ }^{1}$, y, la del colectivo de investigadores, realizadores y autores del Manifiesto Transmedia (2011). De esta forma, se arribó a un total de doce principios, dentro de los cuales se pueden englobar todos de los elementos presentes en las otras propuestas $^{2}$.

A continuación, se buscó evaluar cuantitativamente la eficacia de esta tipología construida para la descripción y caracterización de las narrativas transmedia no-ficcionales. Para ello, se construyó una base de datos conformada por un universo de 216 piezas producidas entre los años 2002 y 2013, constituido por: documentales interactivos, informes multimedia, ensayos fotográficos multimedia, infografías interactivas, así como por otros formatos experimentales como las experiencias multimedia y los docugames.

\footnotetext{
${ }^{1} \mathrm{Al}$ momento de realizar esta integración teórica de los principios transmedia, la tipología propuesta por el MIT Open Documentary Lab e IDFA DocLab constaba de siete principios que posteriormente fueron modificados en parte y ampliados a nueve.

${ }^{2}$ Ver Tabla 1. Anexo A
} 
Para la construcción de la base de datos de la presente investigación se recurrió como fuente primaria a otras bases de datos abiertas disponibles en Internet como la del Laboratorio Abierto de documentales del $\mathrm{MIT}^{3}$; la web de i-Docs ${ }^{4}$, perteneciente al Centro de Investigación en Cultura Digital de la Universidad de West England; y, la del Observatorio del documental interactivo de la Universidad Pompeu Fabra de Barcelona 5 .

El resultado fue una base de datos con 216 casos de estudio delimitados entre los años del 2002 y 2013, constituida por diversas piezas como: documentales interactivos, informes multimedia, ensayos fotográficos interactivos, infografías interactivas y otros formatos experimentales como los docugames y las experiencias multimedia.

Esta base de datos fue diseño de modo tal de poder conformar dos grandes grupos de piezas, uno constituido por 108 piezas multimedia interactivas de carácter informativo y otro por 108 piezas transmedia no-ficcionales. Para la elaboración de estos dos grupos se tuvo en cuenta criterios de semejanza en lo relativo a la procedencia, formato, idioma y año de producción de las piezas seleccionadas.

A continuación estos dos grupos fueron sometidos a un análisis cuantitativo y valorativo para medir la efectividad de los doce principios de la tipología construida, para la caracterización y tipificación de las narrativas transmedia de no-ficción. Este análisis, se hizo con la asistencia del programa informático SPSS con el que se ponderó cada uno de estos casos de estudio siguiendo una escala Bajo-Medio-Alto que se utilizó para valorar el grado de desarrollo del principio en cuestión.

Durante la segunda etapa de investigación titulada "Análisis discursivo de piezas documentales interactivas y otras producciones periodísticas multiplataforma en el marco de una tipología específica para la descripción de las narrativas transmediáticas no-ficcionales", se inició con el análisis cualitativo de los doce principios constituyentes de la tipología construida.

Para ello se planteó la hipótesis de investigación de que las narrativas transmedia son un objeto de estudio bidimensional resultante de la combinación de sus dos elementos constitutivos: "narrativas" y "transmedia". Por lo tanto, su abordaje debe contemplar esta doble naturaleza y analizar como es que se articula el discurso en el plano del contenido, lo que hace a lo "narrativo", así como también indagar como es que se articula el discurso en el plano de la enunciación, lo que hace a su "transmedialidad".

Dada la extensión del estudio, este análisis cualitativo de los principios de la tipología construida se subdividió a su vez en dos fases. En una primera etapa de estudio, realizada durante 2015, se procedió a analizar los 7 principios que hacen al plano de la enunciación: "transmedialidad", "el modelo de negocio", “interfaz", “expansión”, “multiplicidad”, “interactividad” y “participación”; dejando para la

\footnotetext{
${ }^{3}$ Ver: http://docubase.mit.edu

${ }^{4}$ Ver: http://i-docs.org/

${ }^{5}$ Ver: http://inter-doc.org/
} 
segunda fase de investigación, el análisis de los cinco principios restantes que hacen al plano del contenido.

Para esta primera fase del análisis cualitativo, se siguió los postulados de la Teoría de los Discursos Sociales (Verón: 1987, 2013) en lo concerniente a que los discursos pueden analizarse desde tres instancias: producción, circulación y recepción, instancias sobre las que se comprendió los 7 principios de la tipología construida que hacen al plano de la enunciación.

Así la "transmedialidad”, “el modelo de negocio" y la "interfaz" fueron consideradas en función al primer momento de su producción; los principios de "expansión" y "multiplicidad" fueron contemplados en función al segundo momento de su circulación; y por último, la "interactividad" y la "participación” fueron analizados desde el momento de su reconocimiento.

En la segunda y última fase de investigación, realizada durante 2016, titulada "Hacia una narrativa del dato. Análisis cuantitativo y discursivo de diferentes piezas transmedia de no ficción", se buscó analizar desde una perspectiva narratológica la efectividad teórica-empírica de los otros cinco principios restantes que hacen al plano del contenido: "worlbuilding”, "continuidad", "subjetividad", "localización" y "mash-up".

Para ello, de entre el universo de 108 piezas transmedia no-ficcionales se seleccionaron los casos de estudio con mayores niveles de desarrollo del principio en estudio, con el objeto de evaluar la efectividad teórica-empírica del mismo para la caracterización y análisis de piezas transmedia noficcionales

\section{MARCO TEÓRICO}

La presente investigación parte de la concepción teórica de la Media Ecology como marco general para delimitar al fenómeno de las narrativas transmedia. La metáfora de la ecológica aplicada a los sistemas de medios surgió a partir de la década del sesenta acuñada originalmente por Marshall McLuhan y fue posteriormente desarrollada por Neil Postman (2000). La misma postula que la analogía entre el funcionamiento de los medios de comunicación y los ecosistemas, planteando así una ecología de los medios.

Al mismo tiempo, en el contexto de la Media Ecology (Postman, 1970), se postula que estos "ecosistemas" de medios producen modificaciones perceptivas, cognitivas, axiológicas y emotivas a los sujetos de la sociedad en la que funcionan. Esta teoría busca analizar los ambientes considerando su estructura, contenidos e impacto sobre las personas (p.161)

La Media Ecology constituyó también un andamiaje teórico para el desarrollo de la noción de convergencia, posteriormente desarrollada por Henrry Jenkins en su obra Convergence Culture (2008). 
Este concepto es fundamental para entender los efectos de los nuevos medios y plataformas sobre los dispositivos culturales de las diferentes sociedades contemporáneas. La convergencia se entiendo así como el flujo de contenido a través de múltiples plataformas mediáticas, la cooperación entre múltiples industrias mediáticas y el comportamiento migratorio de las audiencias mediáticas (p.14).

Además de permitir apreciar el impacto que la emergencia de estas nuevas tecnologías, la media Ecology aporta también un fecundo marco teórico para analizar el surgimiento, evolución y adaptación de estos nuevos medios a lo largo del tiempo. En este sentido, cabe destacar las contribuciones de Robert K. Logan (2010) que postula que el lenguaje, la cultura, la tecnología y los medios de comunicación se comportan como organismos que viven, evolucionan, se propagan e interactúan unos con otros dentro de un ecosistema de medios determinado (p.1).

Es sobre este aspecto de los medios como organismos que evolucionan que Carlos Scolari (2012) desarrolló su noción de Media Evolution, afirmando que el concepto de evolución crea un marco teórico para el estudio de la historia de los medios de comunicación, a la vez que aporta nuevos conceptos y preguntas acerca de la extinción, la supervivencia, la co-evolución e hibridizaciones de los diferentes medios. (p.1)

Profundizando sobre estos temas, Scolari (2013a) propone un modelo basado en el "ciclo vital de los medios" presentado por Lehman-Wilzig y Cohen-Avigdor (2004) para analizar el surgimiento y evolución de los medios de comunicación. El modelo simplificado de Scolari consta de tres fases: emergencia, dominación y supervivencia/extinción. En la primera fase, la emergencia de una nueva tecnología de comunicación conlleva una serie de desafíos que solo pueden abordarse de una forma posible: prueba y error. En el segundo momento, en analogía con los ecosistemas biológicos, en la ecología de los medios también hay especies dominantes que, durante su época de oro son capaces de imponer su propia dinámica al resto. En la tercera fase, los ecosistemas de medios sufren alteraciones producto de los nuevos inventos tecnológicos, las condiciones económicas, o las prácticas sociales; factores que conllevan a los viejos medios de comunicación a tener que interactuar con los nuevos. Si el viejo medio no pude adaptarse a las nuevas condiciones de la ecología de los medios, corre el riesgo de extinguirse. (p.8-10)

\section{DE LO MULTIMEDIA A LO TRANSMEDIA}

Si bien el término multimedia se remonta a mediados del siglo XX al ámbito de las artes plásticas, para finales de los la década del ochenta y principios de los noventa comenzó a popularizarse a raíz de la aparición de computadoras personales equipadas con placas de video y dispositivos lectores de CD-ROM 
(por su sigla del inglés Compact Disc Read-Only Memory), tecnologías que permitían integrar varios medios a la vez como sonido, texto, animaciones, vídeo, imágenes y música.

Estos desarrollos tecnológicos, constituían lo que a mediados de la década del ochenta autores como R.J. Shavelson, G. Salomon, J. Barker y R. N. Tucker habían caracterizado como "multimedia interactiva", entendida como sistemas digitales en los cuales el usuario tenía control sobre la presentación de los contenidos y podía elegir que ver y oír, algo que en el mundo de los medios analógicos el usuario estaba forzado a experimentar de manera lineal.

Con el advenimiento de Internet a mediados de los noventa, autores como V. Balasubramanian, A. Bianchini, M. Salampasis, R. Rada, P. Diaz, O. Catenacci, I. Aedo comenzaron a hablar de "hipermedia". El término surge de la fusión de los conceptos de "hipertexto" y "multimedia" y puede definirse como: "organización de información textual, visual gráfica y sonora a través de vínculos que crean asociaciones entre información relacionada dentro del sistema" (Caridad y Moscoso, 1991, p.48). Sí bien no deja de ser un tipo específico de multimedia interactiva, podría considerarse como una primera evolución de lo multimedia, producto de su adaptación al entonces nuevo ecosistema de la Word Wide Web.

Desde lo empírico, las diversas producciones multimedia que se han venido produciendo desde mediados de la década del noventa, tanto online como offline, a menudo se pueden clasificar de acuerdo a los géneros a los que pertenecen. Así, comúnmente se distinguen cuatro grandes géneros multimedia: multimedia educativa, multimedia publicitaria, multimedia comercial y multimedia informativa.

La presente investigación circunscribió su accionar a la última de estas categorías, en la que a su vez se incluyen varios subgéneros como: los documentales interactivos, los informes multimedia, las infografías interactivas, los ensayos fotográficos interactivos, así como otros formatos experimentales como los docugames y las experiencias multimedia.

Estos diferentes formatos comenzaron a aparecer a principios de la década del 2000, producidos en mayor medida por los principales diarios online de todo el mundo, así como por realizadores independientes. Con el paso del tiempo y a la incorporación de nuevas plataformas y dispositivos, estos formatos multimedia interactivos de carácter informativo han ido evolucionando, adquiriendo nuevas formas y estructuras narrativas que esta investigación considera adecuadas estudiar y caracterizar a través del paradigma de la transmedialidad.

\section{LA NOCIÓN DE TRANSMEDIALIDAD}

La primera en utilizar el término de transmedialidad en el ámbito de la investigación crítica de los medios de comunicación fue Marsha Kinder (1991) quien acuñó el término "transmedia intertextuality" 
para describir cómo algunas narrativas infantiles se desplazaban a través de múltiples plataformas y permitían a sus públicos diferentes niveles de interacción. En este trabajo, Kinder postula la existencia de "supersistemas" compuestos por la televisión, los videos juegos y el cine que interpelan a los niños no solo a consumir determinados productos, sino que se orientan a reproducir en ellos un determinado modelo de consumo.

Otra de las pioneras en utilizar el término fue la narratóloga Marie Laurel Ryan (2000) quien postuló a la transmedialidad como la primera de ocho leyes para la creación de contenido en el contexto de la convergencia de medios. La autora entiende a la transmedialidad como una estrategia integral de producción. En sintonía con esta concepción sobre la producción de contenido en un contexto de convergencia, el siguiente investigador en emplear el término fue Henry Jenkins (2008), quien postuló que la narración transmedia constituye de por sí una nueva estética, producto de la convergencia de los medias. Al mismo tiempo, las narrativas transmedia plantean nuevas exigencias a los consumidores, que ya no se limitan a consumir pasivamente las historias, sino que por el contrario, participan activamente del desarrollo de las mismas (p.31).

Estos primeros trabajos de Kinder, Ryan y Jenkins pueden considerarse como los fundacionales del campo de la investigación en torno a las narrativas transmedia, delimitando conceptualmente así, un fenómeno mediático que se venía a desarrollando desde hacía ya varias décadas, a veces de manera intuitiva y otras de forma estratégica, en el ámbito de la industria de los contenidos audiovisuales.

Si bien la mayoría de las conceptualizaciones realizadas hasta la fecha en torno a las narrativas transmedia se limitan casi exclusivamente al ámbito de la ficción, cabe destacar las iniciativas de algunos investigadores por ampliar el concepto hacia otras narrativas de carácter no-ficcional, entre los que cabe destacar a Scolari (2013b, 2014), Porto \& Flores (2014), Irigaray \& Lovato (2015), Liuzzi (2015), y Larrondo (2016). Estos aportes teóricos evidencian que el fenómeno transmedia no solo se limita al ámbito de la ficción, sino que también se puede ampliar para estudiar otros géneros no-ficcionales como los documentales y otras producciones periodísticas multiplataforma.

\section{RESULTADOS ANÁLISIS CUANTITATIVOS}

Los datos del análisis cuantitativo evidencian en primer término que la tipología construida durante la primera parte de esta investigación no fue eficaz para la descripción del grupo de 108 piezas multimedia interactivas de carácter informativo analizadas, pero en cambio, sí presentó un alto grado de efectividad para la descripción de las 108 piezas transmedia no-ficcionales analizadas.

Al mismo tiempo, la hipótesis de investigación planteada al principio de la primera fase del trabajo sobre que la narrativas transmedia no-ficcionales pueden ser consideradas como la evolución las piezas 
multimedia interactivas de carácter informativo y que como tales pueden ser reconocidas como un género en sí mismas, se ajusta a los datos obtenidos empíricamente.

En este sentido, cuando se observa la producción de ambos tipos de piezas en función a su tiempo de aparición, se puede apreciar que tanto estas piezas multimedia interactivas de carácter informativo como las piezas transmedia no ficcionales “convivieron" desde el año 2002 hasta el 2013. Sin embargo, se evidencia un declive de la tasa de producción de las primeras, frente a un incremento en la tasa de crecimiento de las segundas conforme al paso del tiempo, en especial a partir del $2007^{6}$.

Al mismo tiempo, se registran aumentos en el número de plataformas utilizadas en las piezas transmedia analizadas a partir del 2007 y del 2010. Este hecho sin duda guarda correlato con la incorporación de los teléfonos inteligentes y la propagación de las redes sociales a partir del 2007, así como con la masificación de las tabletas y televisores inteligentes a partir del 2010.

Con respecto al análisis del modelo de negocio, si bien se observa que tanto las piezas multimedias interactivas de carácter informativo, como las piezas transmedia no-ficcionales analizadas emplean varios tipos de modelos de negocios en sus estrategias de financiación, la tendencia se hace más evidente en las últimas. Así, se pudo identificar un total de ocho modelos de negocios diferentes utilizados para la financiación de la producción de las piezas de estudio transmedia no-ficcionales ${ }^{7}$.

Por último, si bien el principios de "mush-up" mostró que solo pudo aplicarse a la descripción del 13,89\% de las piezas transmedia no-ficcionales analizadas ${ }^{8}$, así como, el principio de "localización” solo pudo aplicarse a la descripción del $50,93 \%$ de los casos de estudio transmedia ${ }^{9}$, los datos señalan que a partir de 2010 en adelante, se registró un crecimiento sostenido de piezas transmedia que incorporaron estos dos principios a sus narrativas.

\section{RESULTADOS ANÁLISIS CUALITATIVOS SOBRE CASOS DE ESTUDIOS}

Siguiendo una segunda hipótesis relativa a que las narrativas transmedia no-ficcionales son un objeto de estudio bidimensional por lo que su abordaje debe contemplar esta doble naturaleza y analizar tanto como es que se articula el discurso en el plano del contenido, tanto como en el plano de la enunciación. Así, en una primera fase de investigación se consideraron los 7 principios de la tipología construida que hacen al plano de la enunciación en función a los tres momentos para el análisis discursivo propuesto en el ámbito de la Teoría de los Discursos Sociales (TDS): producción, circulación y recepción.

\footnotetext{
${ }^{6}$ Ver Grafico 1. Anexo B.

${ }^{7}$ Ver Gráfico 2. Anexo B.

${ }^{8}$ Ver Gráfico 3. Anexo B.

${ }^{9}$ Ver Gráfico 4. Anexo B.
} 
Así, con respecto al primer principio analizado desde la etapa de producción, el de “transmedialidad”, del análisis teórico se desprende que la transmedialidad es una forma particular de configurar los discursos en el actual contexto del sistema de medios contemporáneo, a la par que se la puede conceptualizar como la evolución de la intertextualidad, en primer término, y de la "hipertextualidad", en segundo término.

Asimismo, del análisis empírico del caso de estudio propuesto para el análisis del principio de transmedialidad, el documental interactivo Alma: la hija de la violencia (2012), los resultados obtenidos pusieron en evidencia la utilidad de emplear las categorías "crossmedia" y "transmedia" para la descripción de las diferentes estrategias de producción desplegadas en la generación de un proyecto de esta naturaleza.

En lo referente al segundo principio analizado desde la instancia de producción; el principio de "modelo de negocio", el análisis teórico pudo establecer que dada la denominada Paradoja de la escasez, así como las dos diferentes lógicas productivas comerciales-cerradas y abiertas-colaborativas (Verón, 2013), imperantes en el mercado de los actuales ecosistemas de medios, la instrumentación de cualquier estrategia transmedia siempre requiere por un lado brindar diferentes productos de manera abierta y gratuita, pero al mismo tiempo, necesita del desarrollo de una estructura de negocio que permita su sustento económico.

Reforzando esto, el análisis empírico del universo de estudio y de la muestra representativa de casos seleccionados para el análisis del principio de modelo de negocio, evidencian como todas las diferentes piezas responden tanto a la lógica comercial-cerrada ó abierta-colaborativa. Además, los doce diferentes modelos de negocio identificados confirman lo planteado en la primera etapa de investigación acerca de que la diversidad de modelos de negocios es una característica inherente a las estrategias de producción transmedia.

Con respecto al último de los principios analizados desde la instancia de producción, el de “interfaz", del análisis teórico se pudo establecer que dado el desfasaje inherente a toda semiosis entre producción y recepción, este principio de interfaz se complementa y relaciona directamente en recepción con el principio de interacción. Así, interfaz e interacción pueden entenderse como dos polos constitutivos de un mismo sistema semiótico.

Asimismo, del análisis empírico del caso de estudio propuesto para el análisis del principio de interfaz, el documental interactivo The Sexperience 1000 (2008), se desprendió que las nociones de "contrato de lectura" (Verón, 1985) y de "contrato de interacción" (Scolari, 2009a) son efícaces para describir y analizar las formas en que una determinada interfaz construye su vínculo con el lector/usuario. 
Si bien en el marco de la teoría de los discursos sociales la circulación sólo puede ser definida como diferencia entre la producción por un lado, y las varias modalidades de reconocimiento por el otro, ya que la circulación no deja huellas; dada la naturaleza de los nuevos medios, se considera que sí es posible reconstruir ciertas dimensiones de la circulación social a través de elementos como por ejemplo del análisis de los metadatos.

Con respecto al primer principio analizado desde la instancia de circulación, el de "expansión", del análisis teórico se desprende la correlatividad existente entre los conceptos de "expansión" y “viralización”. Estas nociones implican a su vez, respectivamente la capilaridad social y la replicabilidad que poseen estas narrativas, capaces de conllevar a verdaderos efectos de bola de nieve.

Del análisis empírico del caso de estudio propuesto para el análisis del principio de multiplicidad, el documental interactivo 18 Days in Egypt (2011), se observa que el modelo para graficar la circulación de los discursos planteado por Verón (1993:124-133), así como su posterior readaptación al entorno de los ecosistemas de medios propuesta por Carlón (2018), presentan un alto grado de adecuación para la descripción de este principio de la expansión.

Del estudio teórico del segundo principio analizado desde la instancia de circulación, el de multiplicidad, se deduce que éste debe ser entendida desde la concepción de "modularidad" (Manovich, 2005), categorización que permite analizar la circulación de los discursos dentro de los actuales ecosistemas de medios, a la vez que constituye un marco teórico adecuado para analizar como los elementos discursivos se "encastran" entre sí.

En concordancia con esto, del análisis empírico del caso de estudio propuesto para el análisis del principio de "multiplicidad", el documental interactivo Bar Code (2011), se desprende que el análisis de las cuatro reglas constitutivas de las materias significantes propuesto por Eliseo Verón (1974) se muestra efectivo para la descripción y análisis de los diferentes materialidades que constituyen un producto transmedia ${ }^{10}$.

Con respecto a los dos últimos principios analizados desde la etapa de recepción, se considera que para realizar un estudio y análisis de las gramáticas de recepción en profundidad, hay que recurrir a técnicas de investigación cualitativas como las entrevistas en profundidad, los focus groups, la observación participante; o cuantitativas, como los registro de sesión de navegación, y su posterior análisis a través de mapas de calor, tiempo de exposición, etc.

No obstante, a los fines del presente trabajo de investigación, se consideró que la realización de análisis del tipo exploratorios de los dos principios restantes se muestra suficiente para poder advertir

\footnotetext{
${ }^{10}$ Aquí se plantean algunas diferencias con respecto a lo anteriormente postulado (Astigueta, 2018) en torno a estos últimos dos principios analizados. Se considera que esta reelaboración de los mismos, aporta nuevos elementos que conllevan a un mejor funcionamiento del modelo de análisis propuesto.
} 
diferentes indicios para la caracterización de algunas de las gramáticas de recepción implícitas en las narrativas transmedia no-ficcionales.

Así, del análisis del estudio teórico del primer principio analizado desde la instancia de recepción, el de interactividad, se desprende que así como en producción la noción de contrato de lectura permite analizar la ideología del enunciador, en recepción, conlleva a considerar los diferentes efectos que dicho discursos produce en el enunciatario.

En cuanto al análisis empírico del caso de estudio propuesto para el análisis del principio de interactividad, el documental interactivo The Test Tube (2010), el análisis de los niveles de interactividad propuestos por las diferentes plataformas, permitió inferir diferentes tipologías de usuarios.

En lo relativo al último de los principios analizados desde la etapa de recepción, el de la participación, del análisis teórico se desprende la emergencia de una auténtica cultura de la participación en recepción. La misma se potencia por la multiplicación de nuevos medios y plataformas, que han llevado a una reconfigurado el rol de las audiencias, que ya no se consideran constituidas por consumidores pasivos de discursos, sino por prosumidores, consumidores que producen y contribuyen activamente a la circulación de dichos discursos.

Al mismo tiempo, por el lado análisis empírico del caso de estudio propuesto para el análisis del principio de participación, el documental interactivo Sandy Storyline (2013), el análisis descriptivo de las diferentes alternativas de participación que ofrece el proyecto pone en evidencia los nuevos roles que este tipo de producciones propone a sus usuarios, roles que van desde la financiación, co-producción, difusión, hasta la acción política.

Con respecto a la segunda y última fase de investigación cualitativa relativa a los cinco principios restantes de la tipología construida que hacen al plano del contenido; se destaca, en un primer término, que las diferentes nociones narratológicas utilizadas para el desarrollo y comprensión de los diferentes principios analizados, se muestran eficientes para la descripción y clasificación de los diversos casos de estudio presentados, más allá de haber sido elaboradas varias décadas antes de la aparición de las narrativas transmedia no-ficcionales.

En particular, con respecto al primero de estos cinco principios que hacen al plano del contenido, el de "worldbuilding", el análisis teórico mostró como las nociones de diégesis y mimesis, desarrolladas en el ámbito de la narratología clásica, se revelaron adecuadas para la conceptualización del principio de worldbiuilding, con la peculiaridad de evidenciar que en las narrativas transmedia no-ficcionales, los límites entre las dimensiones diegéticas y miméticas tienden a confundirse.

Asimismo, del análisis empírico del caso de estudio propuesto para el análisis del principio de “worldbuilding”, el documental interactivo Clouds (2013), los resultados obtenidos pusieron en evidencia 
la utilidad de emplear las categorías desarrolladas por Lubomír Doležel (1999), relativas a las cuatro dimensiones constitutivas de los mundos narrativos, para el análisis de este tipo de piezas.

En lo referente al segundo principio analizado desde una perspectiva narratológica el principio de “continuidad”, el análisis teórico mostró como este principio puede ser entendido desde lo que Lev Manovich (2005) denomina la estética de la continuidad, concepción que señala como a partir de la edición digital se pasó de un montaje discontinuo, a un montaje fluido multicapa. Este nuevo tipo de montaje se caracteriza por que sus contenidos se funden en un continuo, que se expresa a través de las diferentes plataformas que constituyen una pieza transmedia.

En este sentido, análisis empírico realizado sobre el documental interactivo Planet Money Makes a T-Shirt (2013), demostró como este principio de continuidad se puede operativizar a través del modelo propuesto por Carlos Scolari (2009b) para identificar las diferentes estrategias narrativas implícitas en este tipo de de producciones transmedia, que a partir de una macro-historia hacen surgir una diversidad de narrativas secundarias.

Con respecto al tercero de los principios analizados desde el plano del contenido, el de "subjetividad", se observó que las nociones de "distancia” y "focalización”, desarrolladas por Gerard Genette (1972) en el ámbito de la narratología durante la década del setenta, presentaron un marco interpretativo adecuado para analizar el principio de subjetividad. En términos generales, podría plantearse que las narrativas transmedia se caracterizan por utilizar diferentes puntos de vista para contar una historia, priorizando las subjetividades tanto de los narradores y personajes, así como la de los usuarios.

Asimismo, del análisis empírico del caso de estudio propuesto para el análisis del principio de subjetividad, el documental interactivo Here at home (2012), se evidenció que el modelo de los cuatro tipos de narradores propuestos por Gerard Genette (1972) en el ámbito de la narratología, se muestra eficaz para la descripción y análisis de las voces narrativas implícitas en las narrativas transmedia.

En cuanto al análisis del cuarto principio a analizar desde el plano del contenido, el principio de la localización, el análisis teórico realizado mostró como la noción de cronotopía desarrollada por Bajtin (1981) puede ser reinterpretada en un sentido más amplio y constituirse así como una categoría de análisis para el principio de localización, señalando las relaciones entre el mundo representado y el mundo real.

En concordancia con esto, el análisis empírico del caso de estudio propuesto para el análisis del principio de localización, el documental interactivo Bear 71 (2012), se comprobó que el modelo de análisis de la interacción entre los diferentes cronotopos (el del autor, el relativo a la obra y el de los receptor) propuesto por el mismo Bajtin (1986), son adecuados para el análisis del cronotopo en piezas transmedia no-ficcionales. 
Con respecto al último de los cinco principios tratados en este trabajo, el principio de mash-up, el análisis teórico concluyó que este principio debe ser conceptualizado como la evolución del concepto de intertextualidad, entendida esta en cuanto a su dimensión narrativa. Al mismo tiempo, el principio de transmedialidad, tratado durante la primera fase de este análisis cualitativo, debe considerarse como la evolución de la intertextualidad en tanto a su dimensión enunciativa.

En cuanto al análisis empírico de la última pieza analizada, el documental interactivo Out of sight (2013), se desprende que el modelo de Duane Merrill (2006) sobre las tres capas constitutivas de las arquitectura de los softwear mash-up (la del proveedor de contenidos, la del sitio y la del web browser cliente) es adecuado para el análisis del principio de mash-up en piezas transmedia no-ficcionales en general.

\section{CONCLUSIONES}

Dado los resultados obtenidos por las diferentes etapas de análisis cuantitativas y cualitativas se puede concluir que los doce principios de la tipología construida para la descripción de las narrativas transmedia no-ficcionales: "transmedialidad", "worldbuilding", "continuidad", "interactividad", “participación”, “subjetividad”, “expansión”, “serialidad”, “mush-up”, “localización”, “interfaz” y "modelo de negocio", presentan un alto grado de adecuación para la descripción, clasificación y análisis de las narrativas transmedia no-ficcionales en general.

Al mismo tiempo, desde el modelo evolutivo de los medios propuesto por Scolari (2013a), estos datos podrían interpretarse como una tendencia creciente hacia una hegemonía de la transmedialidad en cuanto paradigma productivo "dominante" de las piezas no-ficcionales interactivas, frente a una "supervivencia/extinción" del paradigma de la multimedialidad.

Asimismo, la emergencia de la transmedialidad como estrategia de producción, tiene como correlato material la incorporación masiva de diferentes hardware como los teléfonos inteligentes, Smart TV, tabletas, así como de diferentes software como, las redes sociales y las aplicaciones. La aparición de estas nuevas plataformas y dispositivos conllevó a una profunda reconfiguración de los ecosistemas de medios, así como de las prácticas sociales vinculados a los mismos. Alteraciones que a la fecha, recién se están comenzado a vislumbrar.

También, de los sucesivos análisis semiótico-narrativos de los 12 principios de la tipología propuesta sobre los diferentes casos de estudio seleccionados, se desprende la vigencia que los diversos modelos analíticos desarrollados en el ámbito de la sociosemiótica y de la narratología, presentan para la comprensión de los diferentes fenómenos analizados, más allá de que gran parte de esta literatura haya sido publicada varias décadas antes de la aparición de las narrativas transmedia. 
A los fines de ampliar los alcances de esta tipología construida y de sus doce principios constitutivos, sería aconsejable aplicarlos al análisis de otros objetos de estudio que emplean narrativas no-ficcionales y que también articulan sus discursos a través de varias plataformas como es el caso de la comunicación publicitaria, la comunicación política y la comunicación institucional. 


\section{BIBLIOGRAFÍA}

Astigueta, M. (Septiembre 2018) Propuesta para un modelo de análisis de las narrativas transmedia noficcionales. En Marcelo D’Amico (presidente). XVI Encuentro Nacional de Carreras de Comunicación (ENACOM). Universidad Nacional del Centro de la Provincia de Buenos Aires, Buenos Aires, Argentina

Bajtín M. (1986) Problemas literarios y estéticos. La Habana, Ed. Arte y Literatura

Bajtin, M. (1981) Forms of Time and of the Chronotope in the Novel. Notes towards a Historical Poetics. The Dialogical Imagination. Four Essays by M. M. Bakhtin. Austin, University of Texas Press,

Boutaud, J \& Verón, E. (2007). Del sujeto a los actores. La semiótica abierta a las interfaces. Communication, Paris: Lavoisier, Hermès Science.

Caridad, M. \& Moscoso, P. (1991). Los sistemas de hipertexto e hipermedios. Madrid, Pirámide.

Carlón, M. (2018) ¿Cómo seguir? La teoría veroniana y las nuevas condiciones de circulación del sentido. En Escudero, L.; Soto, M.; Traversa, O.; Fabbri, P. La Semiosis Social. Homenaje a Eliseo Veron. Tercera Época. Serie Transformaciones. deSignis 29 (julio-diciembre de 2018). http://dx.doi.org/10.35659/designis.i29p145-155

Doležel, Lubomir (1999) Heterocósmica. Ficción y mundos posibles. Madrid, Arco Libros

García Carrizo, J. \& Heredero Díaz, O. (2015): Propuesta de un modelo genérico de análisis de la estructura de las narrativas transmedia, Revista Icono 14 (13). 260-285. doi: 10.7195/ri14. v13i2.745

Genette, G. (1972) El discurso del relato: ensayo de método. Paris, Editions du Seuil

Gómez, J. (24 de septiembre 2007) Creating Blockbuster Worlds \& Transmedia Production. En Gómez, J. (Moderador). PGA New Media Council. The Society of Illustrators, Nueva York.

Irigaray F \& Lovato A (2015): Producciones transmedia de no ficción. Análisis, experiencias y tecnologías. Rosario: UNR.

Jenkins, H. (2008). Convergence Culture. La cultura de la convergencia de los medios de comunicación. Barcelona: Paidós.

Jenkins, H. (2009). The Revenge of the Origami Unicorn: Seven Principles of Transmedia Storytelling, en Blog Confessions of an Aca-Fan. [en línea] url: http://henryjenkins.org/2009/12/the_revenge_of_the_origami_uni.html.

Kinder, M. (1991). Playing with Power in Movies, Television, and Video Games: From Muppet Babies to Teenage Mutant Ninja Turtles. Los Angeles: University of California Press

Laurel Ryan, M. (2000, Abr. 14) Collision!: Ceating Core Content in a Post-Convergence World. American Institute of Graphic Arts, Nueva York, Estados Unidos. Recuperado de: http://tauzero.com/Brenda_Laurel/Recent_Talks/ContentPostConvergence.html.

Larrondo A (2016): "El relato transmedia y su significación en el periodismo. Una aproximación conceptual y práctica". Trípodos 38: 31-47. 
Lehman Wilzig, S.; Cohen-Avigdor, N, (2004) The natural life cycle of new media evolution: Inter-media struggle for survival in the internet age. New Media \& Society. https://doi.org/10.1177/146144804042524

Liuzzi Á (2015): "El documental interactivo en la era transmedia: de géneros híbridos y nuevos códigos narrativos". Obra Digital 8: 105-135.

Logan, R. K. (2010). The Biological Foundation of Media Ecology. Explorations in Media Ecology, (9). Recuperado de: http://physics.utoronto.ca/people/homepages/logan/BioMedEcol.pdf

Manovich, L. (2005) El Lenguaje de los nuevos medios de comunicación: La imagen en la era digital. Barcelona: Paidós.

Manovich, L. (2007) Understanding Hybrid Media. Recuperado de http://manovich.net /content/04-projects/055-understanding-hybrid-media/52_article_2007.pdf

Merrill, D (2006) Mashups: The new breed of Web App. An Introduction to Mashups. Recuperado de: http://www.ibm.com/developerworks/library/x-mashups/

Postman N. (1970) “The Reformed English Curriculum.” en A Eurich (Eds) High School 1980: The Shape of the Future in American Secondary Education. Nueva York: Pitman Pub. Corp.

Porto Reno, D.; Flores Vivar, J. (2014). Periodismo Transmedia. Editorial Fragua. Madrid.

Postman, N. (2000). The humanism of media ecology. Nueva York: Proceedings of the Media Ecology Association. Recuperado de: http://mediaecology.org/publications/MEA_proceedings/v1/postman01.pdf

Scolari, C. (2009a, noviembre 23). El Ideológico retorno de las interfaces transparentes. Hipermediaciones. Recuperado de http://hipermediaciones.com/2009/11/23/el-ideologico-retorno-delas-interfaces-transparentes/

Scolari, C. (2009b). Transmedia Storytelling: Implicit Consumers, Narrative Worlds, and Branding in Contemporary Media Production. International Journal of Communication (3), 586-606.

Scolari, Carlos A. (2012) Media Ecology: Exploring the Metaphor to Expand the Theory. International Journal of Communication, (22).Recuperado de: http://onlinelibrary.wiley.com/doi/10.1111/j.14682885.2012.01404.x/full

Scolari, C. (2013a) Media Evolution: Emergence, Dominance, Survival and Extinction in the Media Ecology, International Journal of Communication, (7). Recuperado de: http://ijoc.org/index.php/ijoc/article/view/1919/936

Scolari, C. (2013b). Narrativas transmedia. Cuando todos los medios cuentan. Barcelona. Ed. Deusto

Scolari, C. (2013c, Julio 19). Las Narrativas Transmedia en un manifiesto y 11 Twitts. Hipermediaciones. Recuperado de https://hipermediaciones.com/2013/07/19/las-narrativas-transmedia-en-un-manifiesto-y11-tweets/

Scolari, C. (2014). Narrativas transmedia: nuevas formas de comunicar en la 
era digital, en Anuario Ac/e de cultura digital. Madrid, [en línea] url: http://www.accioncultural.es/media/Default\%20Files/activ/2014/Adj/Anuari o_ACE_2014/6Transmedia_CScolari.pdf. pp. 71-81.

Scolari, C. (2015). Prólogo. En: H. Jenkins, J. Green y S. Ford Cultura Transmedia. La creación de contenido de valor en una cultura en red, Barcelona: Gedisa.

Transmedia Manifest: The Future of Storytelling, 2011. http://www.transmedia-manifest.com

Uricchio, W.; Wolozin, S. y K. Edgerton. MIT Open Documentary Lab e IDFA DocLab, (2012) Moments of innovation. Cambridge, disponible en: ttp://momentsofinnovation.mit.edu/ [Accesado el día 16 de mayo de 2013]

Verón, E. (1974). Para una semiología de las operaciones translinguiísticas. Revista Lenguajes (2). Buenos Aires, Nueva Visión.

Verón. E (1985). El análisis del Contrato de Lectura: un nuevo método para los estudios de posicionamiento de los soportes de los media. Les Medias: Experiences, recherches actuelles, aplications. París, IREP.

VERÓN, E. (1993). "El sentido como producción discursiva”, en La semiosis social. Fragmentos de una teoría de la discursividad. Buenos Aires: Gedisa, páginas.

Verón E. (2013). La semiosis social 2: Ideas, momentos, interpretantes. Buenos Aires, Paidós.

\section{Casos de estudio:}

Dewever-Plana, M.; Fougère, I. (2012) Alma: La hija de la violencia. España: Agence VU,Arte,France Info,Rue 89,taz.de,Télérama,Upian. Recuperado de: http://alma.arte.tv/es/

Gee, A.; Bell, A. (2008) The Sexperience 1000. Reino Unido: Channel 4 Producer, Broadcast Now UK. Recuperado de: http://web.archive.org/web/20141125112705/http://sexperienceuk.channel4.com/thesexperience-1000

George, J.; Minard, J. (2013) Clouds. EE.UU.: Levin, G.; Porter, W. Recuperado de: https://www.cloudsdocumentary.com/

Grubbs, W. (2013) Out of Sight. EE.UU.: Pitch Interactive. Recuperado de: http://drones.pitchinteractive.com/

Kenney,C.; Kestenbaum; D.; Goldstein, J.; Jiang, J.; Davis,J.; McCune, M. ;Bui, Q.; Smith, R.; Chace, Z. (2013) Planet Money Makes a T-Shirt. EE.UU.: Blumberg, A.; Boyer, B.; Davis,J.; Amaria,K; National Public Radio. Recuperado de: https://apps.npr.org/tshirt/\#/title

Mehta, J.; Elayat, Y. (2011) 18 days in Egypt. EE:UU: Kickstarter,Sundance New Frontier Story Lab,Tribeca Film Institute New Media Fund. Recuperado de: http://beta.18daysinegypt.com/

Mendes, J; Allison, L. (2012) Bear 71. Canada: Mendes, J; Allison, L. Recuperado de: https://bear71vr.nfb.ca/ 
National Film Board of Canada Digital Team (2012) Here at home. Canda: Mental Health Commission of Canada, National Film Board of Canada. Recuperado de: https://www.nfb.ca/interactive/here_at_home/

Premo,M. ;Falcone, R (2013) Sandy Storyline. EE.UU.: Ariel Kennan,A.; Gottesdiener, L.; Farinha,P.; Porras, T. Recuperado de: https://www.sandystoryline.com/

Suzuki, D. (2010) The Test Tube with David Suzuki. Canada: National Film Board of Canada, The Vacuum Design. Recuperado de: http://testtube.nfb.ca/

Sweeney, H.; Ronez, J. (2011) Bar Code. Canada: Arte, National Film Board of Canada. Recuperado de: https://www.nfb.ca/interactive/bar_code 
ANEXO A

(Tabla 1.)

\begin{tabular}{|c|c|c|c|c|c|c|c|c|c|c|c|c|}
\hline $\begin{array}{l}\text { Princip } \\
\text { io } \\
\text { / } \\
\text { Tipolo } \\
\text { gía }\end{array}$ & $\begin{array}{l}\text { "transmedial } \\
\text { idad" }\end{array}$ & $\begin{array}{l}\text { "worldbuil } \\
\text { ding" }\end{array}$ & "Continuidad" & $\begin{array}{l}\text { "Interactivida } \\
\text { d" }\end{array}$ & $\begin{array}{l}\text { "Participa } \\
\text { ción" }\end{array}$ & $\begin{array}{l}\text { "Subjetivi } \\
\text { dad" }\end{array}$ & $\begin{array}{l}\text { "Expansi } \\
\text { ón" }\end{array}$ & $\begin{array}{l}\text { "Localizac } \\
\text { ión" }\end{array}$ & "Interfaz" & $\begin{array}{l}\text { "Modelo } \\
\text { de } \\
\text { negocio" }\end{array}$ & $\begin{array}{l}\text { "Mu } \\
\text { sh- } \\
\text { up" }\end{array}$ & $\begin{array}{l}\text { "Serialid } \\
\text { ad" }\end{array}$ \\
\hline $\begin{array}{l}\text { Brenda } \\
\text { Lauren }\end{array}$ & $\begin{array}{l}\text { 1. Pensar } \\
\text { transmedia }\end{array}$ & $\begin{array}{l}\text { 2. Crear } \\
\text { ambientes } \\
\text { 3. Diseñar } \\
\text { narrativas } \\
\text { fundaciona } \\
\text { les }\end{array}$ & $"$ & $\begin{array}{l}\text { 4. Proveer } \\
\text { rituales }\end{array}$ & $\begin{array}{l}5 . \\
\text { Generació } \\
\text { n de } \\
\text { comunida } \\
\text { d de } \\
\text { usuarios } \\
6 . \\
\text { Convertir } \\
\text { a la } \\
\text { audiencia } \\
\text { en autores }\end{array}$ & $\begin{array}{l}7 . \\
\text { Facilitar } \\
\text { la } \\
\text { creación } \\
\text { de } \\
\text { identidad } \\
\text { personal }\end{array}$ & $\begin{array}{l}\text { 8. Crear } \\
\text { tratamie } \\
\text { ntos y } \\
\text { escenari } \\
\text { os para } \\
\text { que la } \\
\text { historia } \\
\text { pueda } \\
\text { ser } \\
\text { consumi } \\
\text { da a } \\
\text { través de } \\
\text { diferente } \\
\text { s } \\
\text { dispositi } \\
\text { vos y } \\
\text { contexto } \\
\text { s. }\end{array}$ & & & & & \\
\hline $\begin{array}{l}\text { Henry } \\
\text { Jenkins }\end{array}$ & & $\begin{array}{l}4 . \\
\text { Construcci } \\
\text { ón de } \\
\text { Mundos }\end{array}$ & $\begin{array}{l}\text { 2. Continuidad } \\
\text { vs. } \\
\text { Multiplicidad }\end{array}$ & $\begin{array}{l}\text { 3. Inmersión } \\
\text { vs. } \\
\text { Extrabilidad }\end{array}$ & $\begin{array}{l}7 . \\
\text { Realizació } \\
\mathrm{n}\end{array}$ & $\begin{array}{l}6 . \\
\text { Subjetivid } \\
\text { ad }\end{array}$ & $\begin{array}{l}1 . \\
\text { Expansi } \\
\text { ón vs. } \\
\text { profundi } \\
\text { dad }\end{array}$ & & & & & $\begin{array}{l}5 . \\
\text { Serialida } \\
\text { d: }\end{array}$ \\
\hline
\end{tabular}




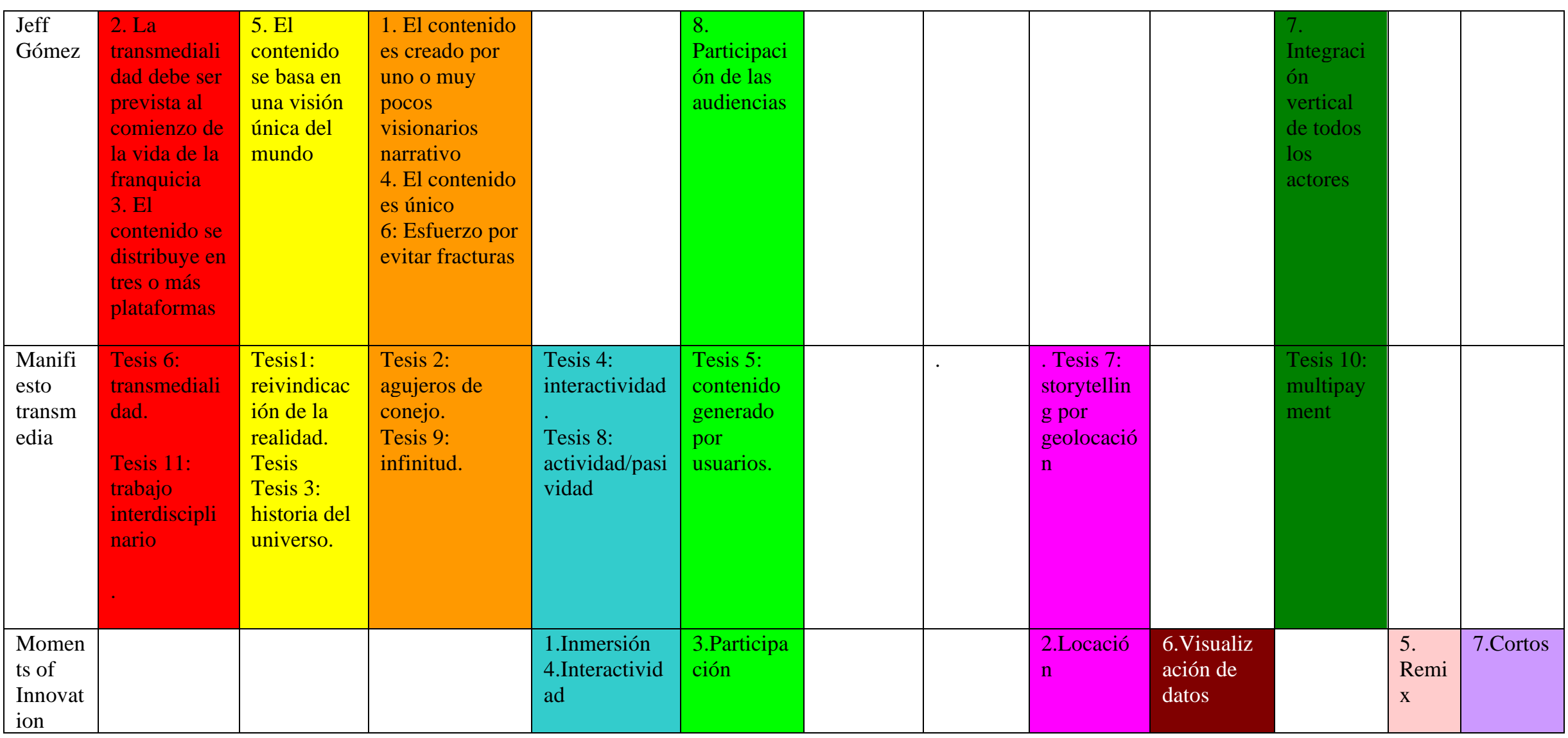




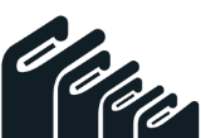

SOUTH FLORIDA

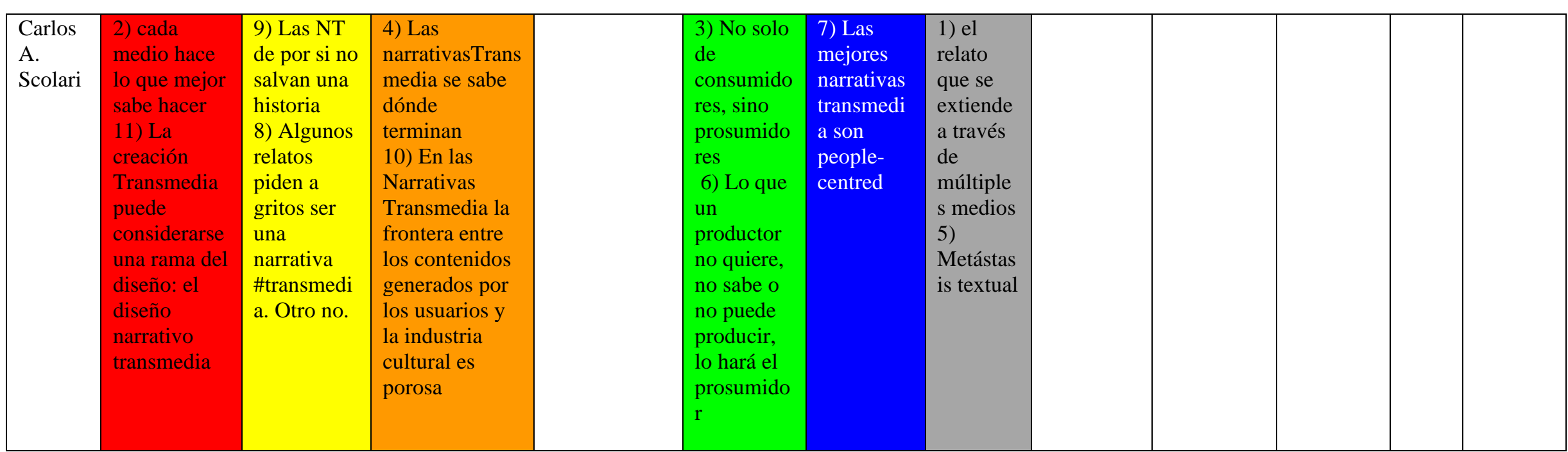




\section{ANEXO B}

(Gráfico 1.)

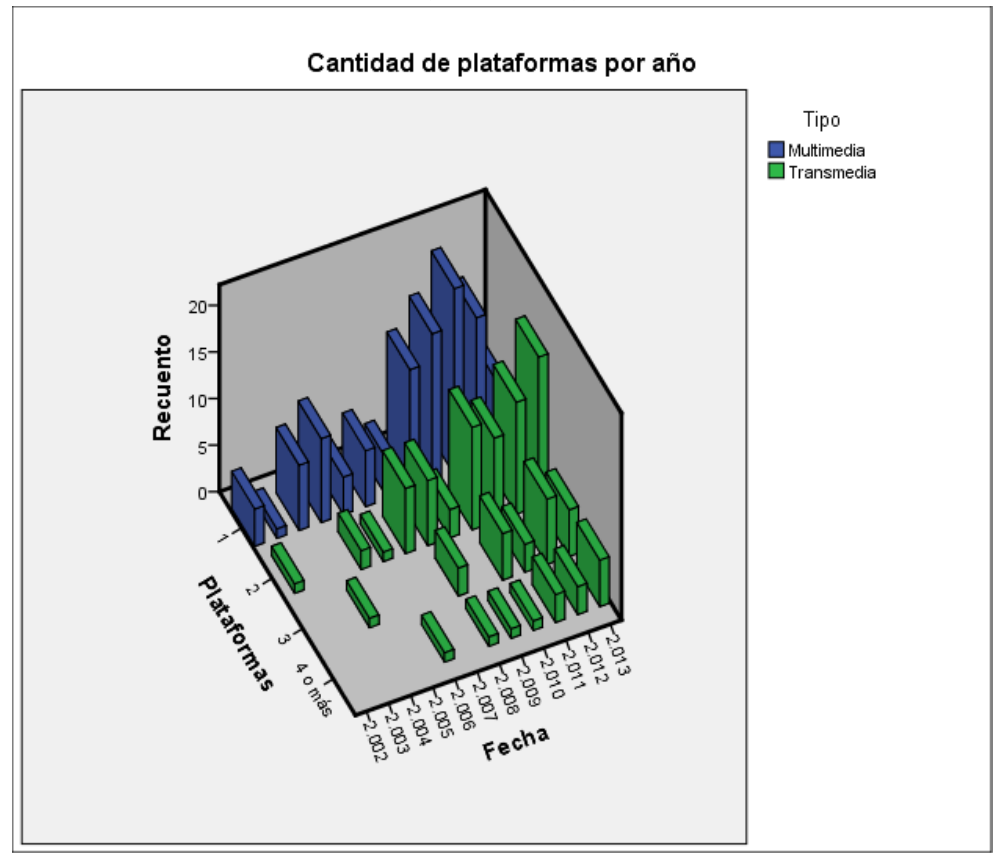

(Gráfico 2.)

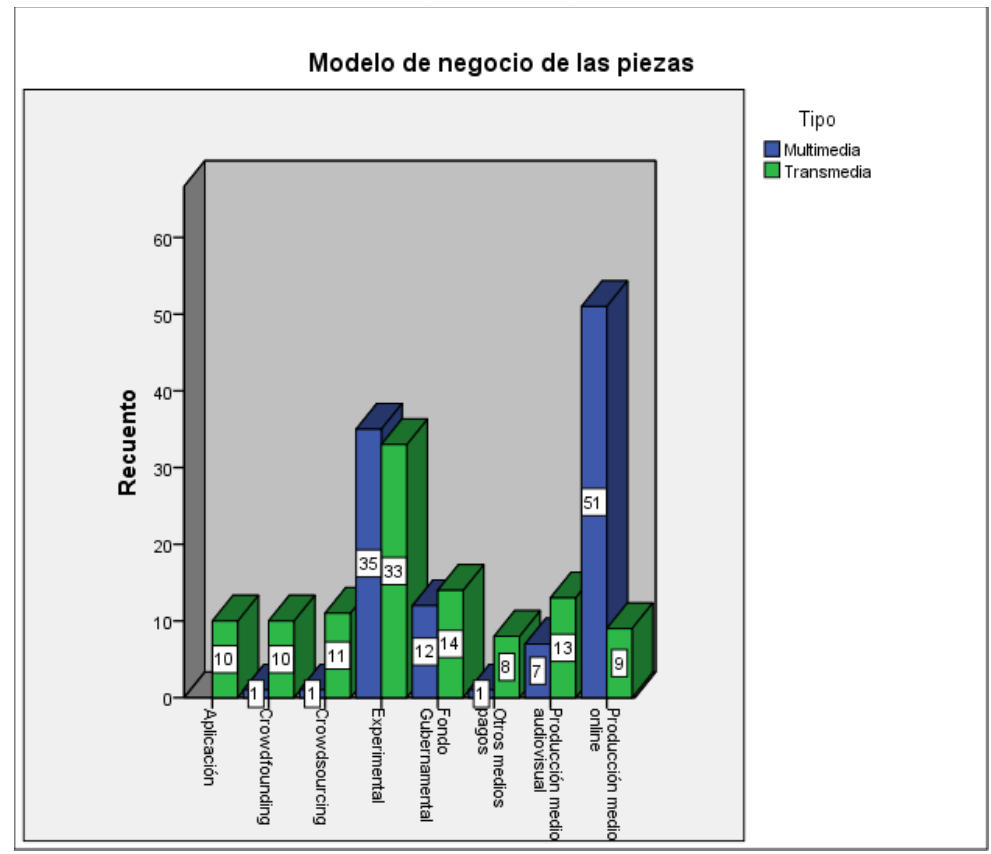


(Gráfico 3.)

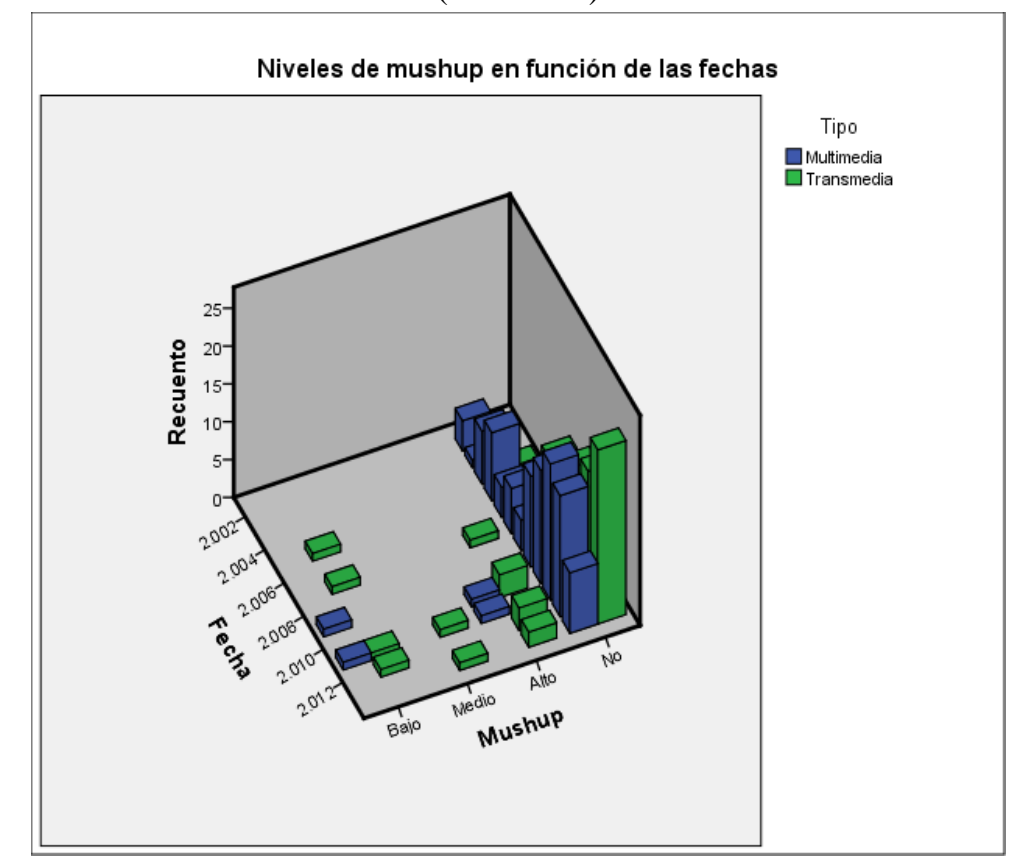

(Gráfico 4.)

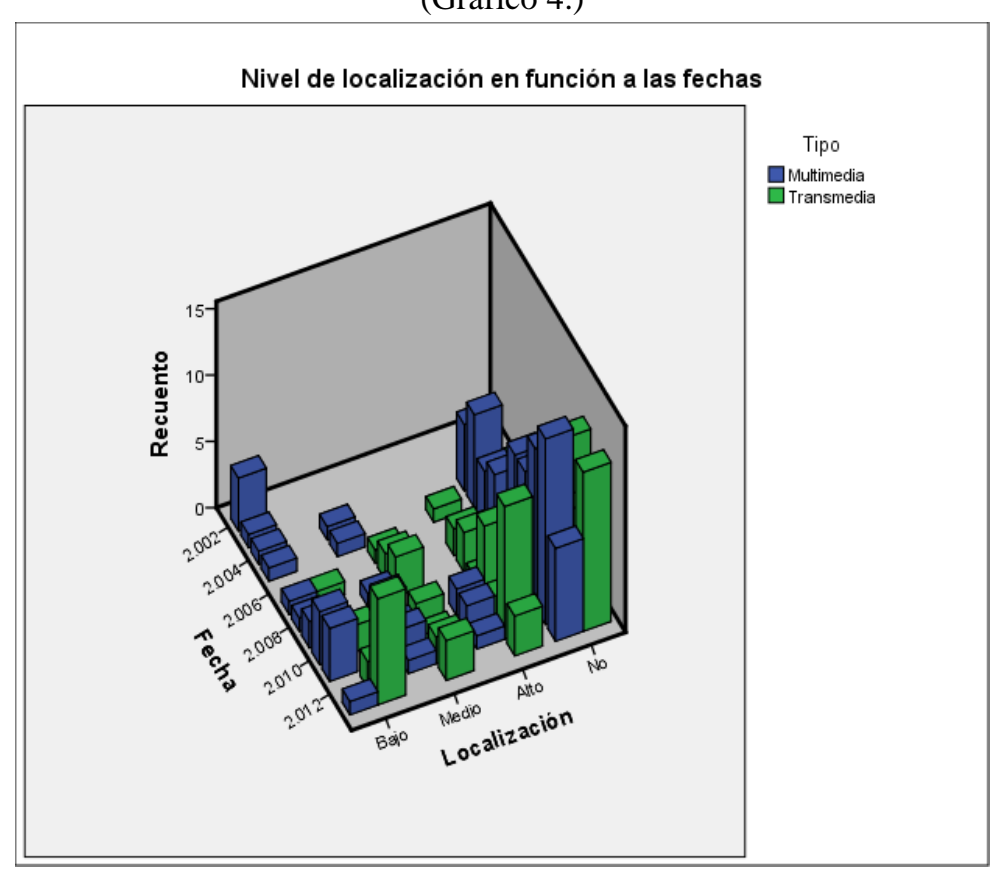

